\title{
INTEGRACIÓN SOCIAL EN ESTUDIANTES UNIVERSITARIOS DE PRIMER AÑO
}

\author{
Yariel Hernández Rosell \\ Facultad de Ciencias de Educación de Ourense \\ yarielhr@gmail.com \\ Leire Pérez Pérez \\ Facultad de Ciencias de Educación de Ourense
}

Fecha de Recepción: 30 Marzo 2019

Fecha de Admisión: 30 Abril 2019

\section{RESUMEN}

La integración social es uno de los componentes fundamentales para el ajuste de los estudiantes a los contextos de la Enseñanza Superior (ES). Este trabajo tiene como objetivo describir la percepción que tienen hombres y mujeres estudiantes universitarios de primer año sobre su integración social al finalizar el primer semestre lectivo. Se aplicó la Escala de Integración Social en la Educación Superior (EISES-R) a 152 estudiantes universitarios de primer año cuyas edades estaban comprendidas entre los 17 y 40 años $(M d n=18.5)$. El $50 \%$ eran hombres y el $50 \%$ mujeres. Tras realizar un análisis de varianza (ANOVA) de un solo factor (sexo), los hombres obtuvieron significativamente mayores puntuaciones medias que las mujeres en equilibrio emocional. Las mujeres obtuvieron puntuaciones significativamente más elevadas que los hombres en las relaciones con la familia. Los resultados encontrados son coherentes con hallazgos previos de la literatura donde se informa que el estado emocional y el apoyo familiar recibido por los estudiantes universitarios tienen un papel importante en su ajuste y bienestar psicosocial y se relacionan con variables tales como la integración en el grupo de iguales, amigos/as, el rendimiento académico y el nivel de aspiraciones. Ambas variables podrían tener un papel significativo diferenciador por sexo como predictores del ajuste y adaptación a la ES, así como en el diseño e implementación de pautas de intervención ante el fracaso 0 abandono en la ES.

Palabras-clave: integración social; estudiantes universitarios de primer año; sexo; ajuste y adaptación; abandono estudiantil

\section{ABSTRACT \\ Social integration in university students of first year. Social integration is one of the funda- mental components for the adjustment of students to the contexts of Higher Education. The objec- tive of this work is to describe the perception that men and women have freshman university stu- dents about their social integration at the end of the first semester. The Scale of Social Integration}




\section{INTEGRACIÓN SOCIAL EN ESTUDIANTES UNIVERSITARIOS DE PRIMER AÑO}

in Higher Education (EISES-R) was applied to 152 first-year university students whose ages were between 17 and 40 years old $(\mathrm{Mdn}=18.5)$. The $50 \%$ were men and $50 \%$ women. After performing an analysis of variance (ANOVA) of a single factor (sex), men obtained significantly higher average scores than women in emotional balance. Women scored significantly higher than men in relationships with the family. The results found are coherent with previous findings in the literature where it is reported that emotional state and family support received by university students have an important role in their adjustment and psychosocial well-being and are related to variables such as integration in the group of equal, friends, academic performance and the level of aspirations. Both variables could have a significant differentiating role by sex as predictors of adjustment and adaptation to higher education, as well as in the design and implementation of intervention guidelines for failure or abandonment in higher education.

Keywords: social integration; freshman university students; gender; adjustment and adaptation; academic abandonment

\section{ANTECEDENTES}

El abandono universitario en España alcanza cifras preocupantes, en torno al 20\% doblando así el objetivo europeo esperado para el año 2020 (MECD, 2015). Algunas investigaciones realizadas en nuestro contexto destacan como las variables más influyentes de dicho abandono las fortalezas de las expectativas, el compromiso académico, la resistencia a la presión, el sentimiento de acompañamiento o la preparación previa a la universidad (Arriaga, Burillo, Carpeño y Casaravilla, 2011). Recientemente también se señala al rendimiento académico previo, la asistencia a clases, la relación con el profesorado o dedicación al estudio como variables implicadas en el fenómeno del abandono (Esteban, Bernardo y Rodríguez-Muñiz, 2016). Tanto unas como otras se vinculan a la realización académica y la integración social apuntados por Tinto, (2006) como componentes integrales del ajuste de los estudiantes a los contextos de Educación Superior (ES). Según su propio modelo de retención estudiantil (Tinto, 1975) los estudiantes emplean la "teoría del intercambio" en la construcción de su integración social y académica. De esta forma, si los beneficios de permanecer en la institución son percibidos por los estudiantes como mayores que los costos personales (esfuerzo y dedicación, entre otros) entonces este permanecerá en la institución. Alternativamente, si se reconocen otras actividades como fuente de mayores recopensas, el estudiante tenderá a desertar. Por ello, la comprensión de las vivencias de los estudiantes en relación con las variables indicadas podrá ayudar a la comprensión del fracaso 0 abandono académico precoz.

Las vivencia o experiencias universitarias se ven matizadas por múltiples variables que influyen en la interacción social de los estudiantes universitarios tales como: actividades universitarias, contacto profesor-estudiante, relaciones entre pares y también importante la relación familia-hijo durante este período.

Numerosos estudios e informes señalan los efectos saludables asociados del contacto estudiantil con los miembros de la facultad (Pascarella y Terenzini, 2005; Tinto, 1993). Las actividades informales de interacción entre estudiantes y profesores, como ser invitado en la casa de un profesor, trabajar en un proyecto de investigación con un miembro de la facultad, hablar con profesores fuera de la clase están positivamente correlacionados con el ajuste del estudiante a la universidad (Kuh, 2003; Kuh y Hu, 2001). Las relaciones con los profesores predijeron el desarrollo de la competencia académica entre los nuevos estudiantes en el primer año de la universidad (Reason, Terenzini y Domingo 2005) y el éxito en términos de satisfacción del segundo año (Graunke y Woosley, 2005; Juillerat, 2000). En conjunto, la persistencia y el éxito de los estudiantes están relacionados con la medida en que los estudiantes interactúan con adultos que apoyan al equipo docente tanto dentro como fuera del aula (Kuh, 2003; Pascarella y Terenzini, 2005). 
Respecto al papel de los iguales, la interacción entre pares determina en buena parte la satisfacción y adaptación de los estudiantes a la vida universitaria. Los estudiantes eligen a sus amigos y pasan tiempo con ellos, lo cual es importante para lo que hacen en la universidad y cómo se sienten con respecto a sus experiencias (Kuh, 1993). Según Astin (1993) los compañeros son la fuente de influencia más potente, que afecta prácticamente a todos los aspectos del desarrollo: cognitivo, afectivo, psicológico y conductual. De hecho, las diferencias en las experiencias de los estudiantes que viven fuera del campus frente a los que viven en residencias universitarias son probablemente las interacciones que tienen además de con los docentes, las que tienen con compañeros y amigos.

Las interacciones entre pares son particularmente importantes en lo que se refiere a la integración social porque los estudiantes tienen más probabilidades de permanecer en la ES cuando se sienten cómodos y conectados con otros estudiantes con intereses y aspiraciones similares (integración social). Las instituciones con niveles más altos de interacción social estudiantil tienen también niveles más altos de aspiraciones educativas de los estudiantes (Pascarella, 1985).

Los estudios también han demostrado que el apoyo (Arriaga et al., 2011) familiar es un importante amortiguador de dificultades durante toda la vida y particularmente durante la transición a la universidad (Álvarez-Pérez y López-Aguilar, 2017). Las relaciones entre padres y alumnos puede servir para mejorar la transición en ES y están relacionadas con el bienestar psicológico, que a su vez está asociado con la adaptación a la Universidad.

Estudios establecen que las percepciones de las relaciones con los padres, es decir; el establecimiento de la mutualidad, el aumento del apoyo social de los padres, más el intercambio con los padres estarán directamente asociados con el bienestar psicológico, que a su vez estará directamente y positivamente relacionado con la adaptación social y el rendimiento académico en el primer año de la universidad (Tinto, 1993). En tal sentido se prevé que la reciprocidad mutua entre padres e hijos mejorará el ajuste general de los estudiantes a la universidad.

El apoyo social parental percibido está relacionado con una nota media elevada superior en la universidad. Cutrona, Cole, Colangelo, Assouline y Russell (1994) demostraron que los estudiantes cuyos padres compartían sus intereses y preocupaciones tenían un mejor desempeño académico y por consiguiente una mejor adaptación en la universidad. Por lo que se plantea que el apoyo social de los padres está relacionado con el rendimiento académico y por consiguiente podría estar relacionado con el ajuste general. En la misma línea Pancer, Pratt, Hunsberger y Alisat (1995) encontraron que la comunicación con los padres estaba indirectamente vinculada a un mejor ajuste universitario.

Wintre y Yaffe (2000) también encontraron que el papel de los padres aportó una contribución pequeña pero importante en la mayoría de los aspectos del ajuste de los hijos a la universidad, observándose efectos diferenciales por género. Por ejemplo, la reciprocidad mutua entre padres e hijos siguió siendo un factor importante en la adaptación masculina. Sin embargo, para las mujeres, la variable paternal significativa fue el inetercanbio de información acerca de la universidad. Este resultado es congruente con los hallazgos de que las mujeres adolescentes tardías tienden más que los hombres a sentirse conectadas con los padres en términos de empatía, comunicación y cercanía (Frank, Avery y Laman, 1988), apoyando la conclusión de que no es simplemente una cuestión de cercanía emocional sino también de cercanía intelectual (Schulthesis y Blustein, 1994).

La satisfacción y el estado emocial de los estudiantes universitarios también juega un papel importante en el ajuste y el bienestar psicosocial de los estudiantes y están relacionadas con variables tales como la integración social en el grupo de iguales, amigos, el rendimiento académico y el nivel de aspiraciones. En diversas investigaciones, se ha comprobado cómo aquellos sujetos con un 


\section{INTEGRACIÓN SOCIAL EN ESTUDIANTES UNIVERSITARIOS DE PRIMER AÑO}

buen equilibrio emocional muestran un mejor ajuste psicosocial (Arrivillaga, Cortés, Goicochea y Lozano, 2003).

Existen factores genéticos y psicosociales que interrelacionados actúan como desencadenantes o mantenedores de estados emocionales negativos como la depresión. Aunque en algunos casos existe un componente genético, en otros, prima la configuración psicológica que el individuo tenga. Con respecto al estudio de estados emocionales negativos en estudiantes universitarios se han encontrado diferencias en cuento al género, pues se observó una relación entre depresión y sexo femenino (Arrivillaga et al., 2003).

Teniendo en cuenta los referentes teóricos anteriormente abordados el presente estudio tiene como principal objetivo describir la percepción que tienen los estudiantes universitarios de primer año sobre su integración social al finalizar el primer semestre lectivo y las diferencias existentes en función del género.

\section{MUESTRA}

La muestra inicial de participantes estaba formada por 186 estudiantes universitarios que cursaban el primer año de los grados de Educación Infantil, Primaria, Informática, Turismo, ADE y Ciencias en el Campus de Ourense. Sus edades estaban comprendidas entre los 17 y 40 años de edad $(M d n=18.0)$. El $41 \%$ eran hombres y el $59 \%$ restante mujeres. Por ámbito de estudio el $66 \%$ cursaba titulaciones pertenecientes al ámbito jurídico-social y el 34\% titulaciones del ámbito científico-tecnológico. Mayoritariamente su procedencia era de carácter urbano $(66 \%)$.

Teniendo en cuenta el objetivo del trabajo y el interés teórico y práctico de la variable género en este tipo de estudios, se equilibró la muestra atendiendo a esa variable. El número de hombres participantes fue de $76(41 \%)$ y se seleccionaron al azar por muestreo polietápico 76 mujeres. Primeramente, se establecieron dos conglomerados de estudiantes en base a la categoría de ser hombre o mujer, después se estratificó uno de los conglomerados proporcionalmente al número de elementos del anterior.

Tras el proceso de selección quedaron 152 participantes con la misma distribución de edad (17 a 40 años) que la muestra inicial ( $M d n=18.5)$. El 50\% eran hombres y el 50\% mujeres. Por ámbito de estudio, el 59\% cursaba titulaciones pertenecientes al ámbito jurídico-social y el $41 \%$ titulaciones del ámbito científico-tecnológico. El 63\% era de procedencia urbana.

\section{Instrumentos}

Se aplicó la Escala de Integración Social en la Educación Superior (EISES-R; Diniz, 2009) que tiene el objetivo de evaluar cómo el estudiante se está adaptando en su nueva vida en la ES. El instrumento cuenta con un total de 30 ítems que integran cinco escalas, cada una con 6 ítems. Una escala hace referencia al equilibrio emocional (EE) integrada por ítems del tipo "Me he sentido decaído/a en este período como estudiante de Educación Superior"; otra escala se refiere a las relaciones con colegas (RC), intregrada por ítems como "Me he sentido decepcionado/a con mis compañeros/as de Educación Superior "; la relación de los estudiantes con los profesores (RP) es otra escala con ítems como "He tenido dificultades para hablar con los/as profesores/as fuera del aula"; una cuarta escala hace referencia a la relación con la familia (RF) y contiene ítems como "Mis padres no entienden los problemas de mi actual vida de estudiante"; y una quinta escala se centra en la relación con lo amigos (RA), con ítems como "Me he sentido decepcionado/a con mis amigos durante mi vida actual como estudiante".

Las respuestas a los ítems se recogieron siguiendo una escala de cinco puntos en formato tipo likert: 5- total acuerdo; 4- de acuerdo; 3- ni de acuerdo ni en desacuerdo; 2- en desacuerdo y 1total desacuerdo. 


\section{Procedimiento}

Los estudiantes, evaluados en los diferentes ámbitos de estudio, fueron seleccionados en función de su heterogeneidad y de la disponibilidad de los profesores. La recogida de los datos la realizaron miembros del equipo de investigación, al inicio y final del $1^{0}$ semestre, dentro de las aulas, en horario académico y a través de la administración colectiva de la Escala de Integración Social en la Educación Superior (EISES-R). Tras proporcionarles información acerca de la naturaleza y objetivos de la investigación, se solicitó a los estudiantes su participación voluntaria, garantizando el anonimato de sus respuestas.

\section{DISEÑO Y ANÁLISIS DE DATOS}

Se realizaron estadísticos descriptivos y análisis de varianza de un solo factor (ANOVA por sexo) para los factores de integración social (EISES-R) y se utilizó el Statistical Package for Social Science (SPSS) en su versión 18.0 para el análisis de los datos.

\section{RESULTADOS}

Los estadísticos descriptivos y resultados del análisis de varianza para la variable género (Tabla 1) mostraron que los hombres y mujeres universitarios de primer año participantes en el estudio se diferenciaron significativamente en su integración social, específicamente en equilibrio emocional y la relación con las familias. Con respecto al equilibrio emocional los hombres obtuvieron una puntuación media significativamente más elevada que las mujeres. En la relación con la familia fueron las mujeres las que obtuvieron una puntuación media significativamente más elevada que los hombres (Tabla 1).

Tabla 1

Estadísticos descriptivos para los factores de integración social por género

\begin{tabular}{|c|c|c|c|c|c|c|}
\hline \multirow[t]{2}{*}{ Factores } & \multicolumn{2}{|c|}{ Hombres $n=76$} & \multicolumn{2}{|c|}{$\begin{array}{c}\text { Mujeres } \\
n=76\end{array}$} & \multirow[b]{2}{*}{$F^{I}$} & \multirow[b]{2}{*}{$p$} \\
\hline & $M$ & $D T$ & $M$ & $D T$ & & \\
\hline $\begin{array}{l}\text { Integración } \quad \text { social } \\
\text { (EISES-R) }\end{array}$ & & & & & & .013 \\
\hline Equilibrio emocional & 22.22 & 5.59 & 19.76 & 6.45 & 6.324 & \\
\hline $\begin{array}{l}\text { Relación } \\
\text { compañeros/as }\end{array}$ & 23.95 & 4.06 & 23.97 & 4.54 & .001 & .970 \\
\hline $\begin{array}{l}\text { Relación } \\
\text { profesores/as }\end{array}$ & 23.21 & 5.67 & 22.09 & 4.60 & 1.784 & .184 \\
\hline Relación familia & 20.87 & 4.86 & 22.87 & 4.67 & 6.687 & .011 \\
\hline Relación amigos/as & 23.70 & 3.67 & 24.58 & 4.81 & 1.614 & .206 \\
\hline
\end{tabular}

${ }^{\mathrm{I}} g l(1,151)$

\section{DISCUSIÓN Y CONCLUSIONES}

El estudio tiene como objetivo describir la percepción que tienen los estudiantes universitarios de primer año sobre su integración social al finalizar el primer semestre lectivo, suponiendo diferencias significativas en función género. 


\section{INTEGRACIÓN SOCIAL EN ESTUDIANTES UNIVERSITARIOS DE PRIMER AÑO}

Se evidenciaron diferencias estadísticamente significativas entre hombres y mujeres en cuanto a su percepción sobre equilibrio emocional y apoyo familiar recibido. Los hombres se perciben con más equilibrio emocional que las mujeres mientras que estas perciben un mayor apoyo familiar con respecto a los hombres.

En este trabajo se han obtenido resultados que van en línea con los estudios que confirman que las mujeres perciben un mayor apoyo familiar durante su integración social a la educación superior, debido a que tienden a sentirse más conectadas con los padres en términos de empatía, comunicación y cercanía con respecto a los hombres. En este sentido se podría interpretar que las mujeres tendrían un mayor nivel de adaptación social debido a la percepción de reciprocidad emocional con sus progenitores (Tinto, 1993). Más allá de los aspectos emocionales, la cercanía intelectual es también un factor relevante en la explicación de la adptación a la ES por parte de las mujeres (Schulthesis y Blustein, 1994). Esto indica que el papel de las relaciones parentales puede ser un predictor del ajuste de las mujeres a la universidad (Pancer, Pratt, Hunsberger y Alisat, 1995).

En equilibrio emocional los hombres obtuvieron puntuaciones superiores que las mujeres. Este resultado coincide con algunos estudios que muestran que existe una relación entre los estados emocionales negativos (depresión) y las mujeres estudiantes universitarias (Arrivillaga et al., 2003). La medida del factor de equilibrio emocional de la escala utilizada en este estudio está muy orientada hacia la detección de estados emocionales de decaimiento, depresión, descontrol e irritación, lo que justificaría este resultado; extrapolándose además un mayor ajuste psicosocial por parte de los hombres que de las mujeres (Arrivillaga et al., 2003).

A modo de conclusión, estos factores de integración social podrían tener un papel significativo diferenciador entre hombres y mujeres como predictores en el ajuste y adaptación social de los estudiantes a la ES.

Los resultados evidenciados en este trabajo necesitan ser replicados en muestras de mayor tamaño y diversidad para obtener un mejor nivel de validez interna y externa. En ese mismo sentido se deberían seleccionar los participantes al azar y aplicarles instrumentos de medida de intregración social donde pudiesen analizarse conductas manifiestas.

Atender a estas cuestiones de validez perimitirían realizar el diseño e implementación de propuestas de intervención en función del género para evitar, en último término el fracaso o abandono en la ES.

\section{REFERENCIAS BIBLIOGRÁFICAS}

Álvarez-Pérez, P. y López-Aguilar, D. (2017). Recursos de orientación para la transición académica y la toma de decisiones del alumnado: el programa "Universitarios por un día". Revista de Innovación Docente Universitaria. RIDU, (9), p. 26-38.

Arriaga, J., Burillo, V., Carpeño, A. y Casaravilla, A. (2011). Caracterización de los tipos de abandono. Dividamos el problema y venceremos fácilmente. I Conferencia Latinoamericana sobre el Abandono en la Enseñanza Superior, Nicaragua.

Arrivillaga, M., Cortés, C., Goicochea, V.L y Lozano, T.Ma. (2003). Caracterización de la depresión en jóvenes universitarios. Univ. Psychol. Bogotá (Colombia) 3 (1), 17-26.

Astin, A. W. (1993). What matters in college? four critical years revisited (1st Ed.). San Francisco: Jossey-Bass.

Cutrona, C. E., Cole, V., Colangelo, N., Assouline, S. G., and Russell, D. W. (1994). Perceived parental social support and academic achievement: An attachment theory perspective. Journal of Personality and Social Psychology, 66, 369-378.

Esteban M., Bernardo A. y Rodríguez-Muñiz, L.J. (2016) Permanencia en la universidad: la importancia de un buen comienzo. Aula Abierta, 44, pp. 1-6. 
Frank, S. J., Avery, C. B and Laman, M. S. (1988). Late adolescents'perceptions of their relationships with their parents: Individual differences in connectedness, competence, and emotional autonomy. Developmental Psychology, 24, 729-737.

Graunke, S. S and Woosley, S. A (2005). An exploration of the factors that affect the academic success of college sophomores. College Student Journal, 39(2): 367-377.

Juillerat, S. (2000). Assessing the expectations and satisfactions of sophomores. In visible solutions for invisible students: helping sophomores succeed (Monograph 31), edited by L. A. Schreiner and J. Pattengale, 19-29. Columbia, SC: University of South Carolina, National Resource Center for the First-Year Experience and Students in Transition.

Kuh, G. D. (1993). In their own words: what students learn outside the classroom. American Educational Research Journal, 30(2): 277-304.

Kuh, G. D., and Hu, S. (2001). The effects of student-faculty interaction in the 1990s. Review of Higher Education, 24(3): 309-332.

Kuh, G. D. (2003). What we're learning about student engagement from NSSE: benchmarks for effective educational practices. Change, 35(2): 24-32.

MECD. (2015). Datos básicos del sistema universitario español. Curso 2014-2015. Recuperado de http://bit.ly/2klsC70.

Pancer, S. M., Pratt, M., Hunsberger, B. y Alisat, S (1995). Great expectations:Parent-child discussion and the impact of pre-university expectations on adolescent transitionto university. Paper presented at the biennial meeting of the Society for Research in Child Development, Indianapolis, IN.

Pascarella, E. T. (1985). College environmental influences on learning and cognitive development: a critical review and synthesis. In higher education: handbook of theory and research, Vol. 1, edited by J. C. Smart, 1-62. New York: Agathon

Pascarella, E .T. and Terenzini, P. T. (2005). How college affects students: a third ecade of research. San Francisco: Jossey-Bass.

Reason, R. D., Terenzini, P. T., and Domingo, R. J. (2005). First things first: developing academic competence in the first year of college. Paper presented at the annual meeting for the Association for Institutional Research, San Diego, CA.

Schulthesis, D.E.P., and Blustein, D. L. (1994). Role of adolescent-parent relationships in college student development and adjustment. Journal of Consulting Psychology, 41, 248-255.

Tinto, V. (1975). Dropout from higher education: a theoretical synthesis of recent research. Review of Educational Research, 45: 89-125.

Tinto, V. (1993). Leaving college: rethinking the causes and cures of student attrition. (2nd ed.).Chicago: University of Chicago Press.

Tinto, V. (2006). Retention: What Next? Journal College Student Retention, 8 (1), 1-19.

Wintre, M.G., and Yaffe, M. (2000). Firts-years student s adjustment to university life as a function of relationships whih parents. Journal of Adolescent Research, 15(1): 9-37. 
